\title{
Differenzierung pflegerischer Aufgaben in der Psychiatrie durch neue Berufsbilder und Zusatzausbildungen
}

\author{
Differentiation of Nursing Tasks in Psychiatric Wards through New Professions \\ and Additional Training
}

Autoren

Pro: Kai G. Kahl, Stefan-M. Bartusch, Martin Greetfeld

Kontra: Michael Schulz

Bibliografie

DOI 10.1055/s-0029-1220344

Psychiat Prax 2009; 36:

208-210

(c) Georg Thieme Verlag KG

Stuttgart · New York .

ISSN 0303-4259

Korrespondenzadressen

PD Dr. med. Kai G. Kahl

Klinik für Psychiatrie, Sozial-

psychiatrie und Psychotherapie

Medizinische Hochschule

Hannover (MHH)

Carl-Neuberg-Straße 1

30625 Hannover

kahl.kai@mh-hannover.de

Dr. rer. medic. Michael Schulz Psychiatrische Pflegeforschung Zentrum für Psychiatrie und Psychotherapeutische Medizin Evangelisches Krankenhaus Remterweg 69-71

33617 Bielefeld

Michael.Schulz@evkb.de

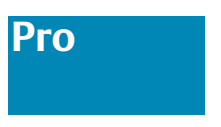

Das Gesundheitswesen ist wegen der zunehmenden Unterfinanzierung der Sozialversicherungen unter erheblichen Effizienzdruck geraten. Folgen dieser Entwicklung sind auf einer übergeordneten Ebene u.a. die Deregulierung und Ökonomisierung der sozialen Sicherungssysteme, der Trend zur Privatisierung von Krankenhäusern und der Versuch, medizinische Leistungen bundeseinheitlich qualitativ und ökonomisch vergleichbar zu machen. Speziell die Einführung der DRGs hat in den somatischen Fächern zu einer Spezialisierung und überregionalen Zentrenbildung geführt, die geradezu gegenläufig ist zu dem Anspruch einer gemeindenahen, umfassenden Versorgung psychisch Kranker.

Auf der Mitarbeiterebene belastet die Verdichtung der Arbeitsabläufe bei gleichzeitiger Pflicht zur Einhaltung leitliniengerechter Therapie das berufliche und persönliche Selbstverständnis der Akteure. Die Arbeit am Patienten - Kernstück der „sprechenden Medizin“ - kommt daher in unserem Fachgebiet häufig zu kurz. Es wäre allerdings einseitig, die Versorgungsprobleme in psychiatrischen und psychotherapeutischen Krankenhäusern ausschließlich als Folgen einer zunehmenden Ökonomisierung darzustellen. Viele „Zeitfresser“ im psychiatrisch-psychotherapeutischen Alltag sind auf Probleme in der Aufgabenund Prozessorganisation zurückzuführen. Einer (von mehreren) Auswegen aus dieser Situation könnte in der Differenzierung pflegerischer Aufgaben in der Psychiatrie liegen, beispielsweise durch die Einführung eines neuen Berufsbildes im Rahmen einer Zusatzqualifikation.

Die umfassende Behandlung psychisch Kranker erfordert einen verhältnismäßig hohen Aufwand an Personal aus unterschiedlichen Berufsgruppen. In der Regel müssen multiprofessionelle Teams zusammenarbeiten, um unterschiedliche Ebenen von Krankheitsfaktoren zu behandeln. Im Idealfall ergibt sich eine Therapie entlang des „biopsychosozialen“ Krankheitsmodells, in der die biologischen, psychologischen und sozialen Einflussgrößen auf das Krankheitsgeschehen beeinflusst werden [1].

In der Praxis sind das Ausrichten des therapeutischen Handelns nach dem „biopsychosozialen“ Behandlungsmodell und das dazu nötige „multiprofessionelle Team“ häufig ein schwer zu erreichendes Ideal. Hierfür gibt es viele Gründe: Die unterschiedlichen Berufsgruppen haben in der Behandlung psychisch Kranker unterschiedliche Behandlungsziele, die sich nicht immer ergänzen. So sind beispielsweise die Pflegeprozesse an den Aktivitäten des täglichen Lebens (ATL) ausgerichtet, ärztliche Prozesse beispielsweise an der Optimierung einer Psychopharmakotherapie, die psychotherapeutischen Prozesse schulenabhängig unterschiedlich.

Neben der häufig mangelnden berufsgruppenübergreifenden Verzahnung therapeutischer Maßnahmen sind die Ursachen für das „nicht professionelle Miteinander" vielschichtig: mangelnde Standardisierung von Diagnostik und Therapie, unpassende räumliche Voraussetzungen, unzureichende Absprachen, unklare Tätigkeitsprofile und eine mangelnde Rechtssicherheit in Bezug auf das Berufs- und Dienstrecht (u.a. der „Arztvorbehalt“) [2]. Dies führt zu Situationen, in denen auch motivierte Mitarbeiter die Orientierung verlieren und sich notwendigen therapeutischen und ökonomischen Anpassungsprozessen entziehen. Darüber hinaus ist unter diesen Umständen die Umsetzung einer leitliniengerechten Therapie erschwert.

\section{Ein Lösungsvorschlag: Übertragung} therapeutischer Aufgaben durch eine qualifizierte Zusatzausbildung

Ein wesentlicher Aspekt der therapeutischen Arbeit liegt darin, den Patienten zum „Experten in eigener Sache“ zu machen. Das umfassende Wissen um die eigene Erkrankung und deren Be- 
handlungsmöglichkeiten fördert die Bereitschaft, gemeinsam erarbeitete Therapievorschläge langfristig einzuhalten. Die Einhaltung therapeutischer Empfehlungen auch nach Abklingen der akuten Symptomatik ist abhängig von der Akzeptanz durch die Patienten, die durch psychoedukative Gruppen- und Angehörigenarbeit verbessert werden kann.

In der Praxis stößt die Einführung von Angehörigengruppen und psychoedukativen Gruppen häufig an personelle, inhaltliche und rechtliche Grenzen. Diese Situation ließe sich durch eine Differenzierung pflegerischer Tätigkeit verbessern, indem die pflegerischen Aufgaben in qualifizierte Fachpflege, psychiatrische Grundpflege und Basispflege sowie pflegeferne Aufgabenbereiche eingeteilt werden. Die Fachpflege hat in diesem Modell die Aufgabe, psychoedukative und soziotherapeutische Gruppenangebote sowie Angehörigengruppen eigenverantwortlich unter Supervision durchzuführen. Zudem unterstützt der/die Fachpfleger/-in Patienten und Patientinnen* bei der Durchführung spezieller Trainings und Einübung neuer Fertigkeiten (z.B. Skillstraining zur Behandlung von Patienten mit Borderline-Persönlichkeitstörung).

$\mathrm{Zu}$ den Aufgaben der psychiatrischen Grundpflege gehören alle patientennahen und stationsbezogenen Tätigkeiten von der Aufnahme bis zur Entlassung, mancherorts unterstützt durch die Tätigkeit von Krankenpflegehelfer/-innen. Zeitintensive und kernprozessferne Tätigkeiten könnten durch entsprechend qualifiziertes Personal durchgeführt werden. Hierzu kann es ebenfalls notwendig sein, neue Berufsbilder zu prägen. Dies führt zu einer zeitlichen Entlastung von therapeutischem Personal und zu einer Verbesserung der patientenzentrierten Arbeit.

Erforderlich zur Umsetzung dieses Modells wäre die verbindliche Einführung einer qualifizierten Weiterbildung zur/m psychiatrischen Fachpflegerin/ Fachpfleger (Beispiel: Zusatzausbildung zur „Fachkraft für sozialpsychiatrische Betreuung“ an der Medizinischen Hochschule Hannover). In dieser Weiterbildung könnte die Basis für die selbstständige Durchführung von psychoedukativen und soziotherapeutischen Gruppen gelegt werden. Durch eine entsprechende Gestaltung der Lehrpläne könnten die Basisfertigkeiten zur Durchführung von Patienten- und Angehörigengruppen und psychotherapeutische Basiskompetenzen vermittelt werden. Ein praxisorientiertes Examen, in welchem beispielsweise die Durchführung eines Gruppenangebots kollegial überprüft wird, könnte diesen Weiterbildungsgang abschließen.

Wichtig bleibt bei solchen Bemühungen, den therapeutischen Spielraum für jede Berufsgruppe festzulegen. So müssen beispielsweise Fragen der Delegierbarkeit ärztlicher Tätigkeiten und der Berufsgruppenidentität geklärt werden, bevor zuvor ärztliche Tätigkeiten auf andere Berufsgruppen übertragen werden. Diese Diskussion ist in zweifacher Hinsicht relevant: Zum einen müssen unter berufspolitischen Gesichtspunkten klare Zuständigkeiten und Abgrenzungen der therapeutischen Berufsgruppen definiert werden. Weiter muss unter ökonomischen Gesichtspunkten zumindest die Gefahr gesehen werden, dass Arbeitgeber versucht sein könnten, durch Übertragung ärztlicher Tätigkeit an nichtärztliche Berufsgruppen Kosten zu sparen.

\section{Zusammenfassung}

Die „sprechende Medizin“ gerät durch Ökonomisierung, Mitarbeitermangel und Konkurrenz in eine schwierige Situation. Einige Probleme können möglicherweise durch eine Differenzierung von pflegerischen Aufgaben gemildert werden. Notwendig wäre eine verbindliche Einführung von qualifizierten Zusatzausbildungsgängen, die u.a. Kompetenzen in der Durchführung psychoedukativer und soziotherapeutischer Gruppenangebote vermitteln. Eine Definition von Kernprozessen und die Standardisierung von Therapieprozessen sind wichtig, um zu einer möglichst hohen Adhärenz an eine leitliniengerechte Therapie zu gelangen.

Vorhandene Personalressourcen könnten durch eine Übertragung differenzierter therapeutischer Aufgaben an entsprechend qualifiziertes Personal effizienter eingesetzt werden. Die Praxis zeigt, dass hierbei die Patientenzufriedenheit und die Mitarbeiterzufriedenheit gleichermaßen wachsen. So könnte im Idealfall eine intensivierte Therapie im Sinne eines „biopsychosozialen“ Krankheitsmodells ohne Mehrkosten angeboten werden.

\section{Kontra}

Kahl et al. werfen in ihren Ausführungen die Frage nach Kernprozessen in psychiatrischen Krankenhäusern auf und machen Vorschläge zu einer „kernprozessorientierten Tätigkeitsbeschreibung“. Diese Überlegungen möchte ich im Folgenden aus pflegewissenschaftlicher Sicht kommentieren und den Vorschlägen das Konzept eines kooperativen Prozessmanagements entgegenstellen. Die Autoren beklagen zunächst unterschiedliche Behandlungsziele der beteiligten Professionen. Hierzu ist anzumerken, dass spätestens seit der Psychiatrieenquete, vergleichbar zu anderen Disziplinen im Krankenhaus aus gutem Grund mehrere Berufsgruppen am Behandlungsprozess beteiligt sind. Verschiedene Professionen bringen unterschiedliche Blickwinkel und Expertisen ein. Das Zusammenwirken im interprofessionellen Team ist mehr als die Summe der einzelnen Teile.

Die Beteiligung mehrerer Berufsgruppen führt jedoch, wie auch aus den Zeilen des Autors zu lesen ist, zu einer steigenden Komplexität. Die Aufgabe für eine bedarfsgerechte stationäre Behandlung besteht also darin, die unterschiedlichen Teilziele einzelner am Behandlungsprozess beteiligter Professionen unter einem Gesamtziel bzw. in einem Gesamtkonzept zu vereinigen. Dies erfordert aber, dass psychiatrische Kliniken vor die Beschreibung ihrer Kernprozesse Klarheit über das „Produkt“ bzw. das übergeordnete Ziel der Behandlung gewinnen. Solche Ziele könnten z.B. lauten: stärkere patientenzentrierte Versorgung, Förderung der sozialen Inklusion, Befähigung zu langfristigem Krankheitsmanagement oder psychosoziale Versorgung. In einem richtungsweisenden Artikel haben Juckel und Morosini [3] darauf hingewiesen, dass psychiatrische Behandlungsstrategien in Zukunft weniger im Hinblick auf Symptomreduktion dafür aber mehr hinsichtlich der erzielten Verbesserungen im funktionellen und psychosozialen Bereich zu bewerten sind.

Folgt man Kahl et al., dann liegen die Zielsetzungen der Pflege im Trend der Zeit, zielen doch die Interventionen auf „Aktivitäten des täglichen Lebens ab“. Sehr viel unwohler würde ich mich als Arzt mit dem Behandlungsziel „Optimierung der Psychopharmakotherapie“ fühlen. Angesichts der Debatten um Recovery [4] oder der von Weinmann vertretenen kritischen Position gegenüber dem „Erfolgsmythos Psychopharmaka“ [5] sollte Medizin ihre Interventionspalette unbedingt hin zu mehr sprechenden Behandlungsstrategien erweitern. Im Hinblick auf die pharmakologische Behandlung von Depressionen fragt Stefan Weinmann in diesem Zusammenhang: „Was bleibt in der antidepressiven Routinebehandlung mit ihrer steigenden medizinischen Inanspruchnahme und dem zunehmenden Zeitdruck, wenn An- 
tidepressiva problematisiert werden? Was können Psychiater dann noch?"

Heute ist Medikation die am häufigsten in der Psychiatrie zur Anwendung kommende Intervention. Weil die Autoren den Bereich der „Pharmakotherapie“ dem Stations- und Assistenzarzt zugewiesen haben, möchte ich anhand dieses Beispiels deutlich machen, dass es neben den oben angesprochenen berufseigenen Expertisen gerade in der Psychiatrie auch immer zu Schnittmengen kommt. Die Auswahl der richtigen Präparate fällt (im Unterschied zum angloamerikanischen Raum oder zu Holland) in den alleinigen Verantwortungsbereich des Arztes. Gleichwohl bringt es der Klinikalltag mit sich, dass gerade die Berufsgruppe der Pflege in vielfältiger Weise mit der „Pharmakotherapie“ in Berührung kommt. Dies geschieht explizit z.B. durch Einbindung in Psychoedukationsprogramme oder das Herrichten von Medikamenten, durch die Beobachtung von Wirkung und Nebenwirkung oder gar durch die Verabreichung von Zwangsmedikationen. Gleichwohl ist die Thematik der Adhärenz wesentlich komplexer und erfordert spezifische Zugänge. Erfolgreich konnten wir Pflegende in der Durchführung der Adhärenztherapie schulen, einer psychotherapeutischen Kurzintervention inklusive Hausbesuchen nach Entlassung. So lässt sich das Problem des langfristigen Krankheitsmanagements adäquat bearbeiten [6].

Folgt man dem Bericht des Sachverständigenrates zur Begutachtung der Entwicklung im Gesundheitswesen, dann sind hier vor allem folgende Forderungen durch psychiatrische Kliniken zu berücksichtigen: So schlägt das Gremium eine Neudefinition der Rollen und Aufgaben verschiedener Berufsgruppen im Gesundheitswesen vor, um so eine Basis für eine Kultur der Kooperation zu schaffen. Hausner et al. [7] weisen darauf hin, dass die dort genannten Forderungen nach multidisziplinären therapeutischen Strategien mit einer flachen Hierarchie zwischen den Berufsgruppen genau der Arbeitsorganisation entspricht, wie sie in der modernen Psychiatrie angesprochen wird.

Pflege ist Teil der multidisziplinären therapeutischen Strategie, macht also auch Therapie - ähnlich wie die Ergotherapie. Dies macht unbedingt Sinn, denn Dahlgaard u. Stratmeyer [8] folgend, wird dieses Potenzial zu wenig genutzt, was gerade aus ökonomischer Sicht nicht zu akzeptieren ist. Pflege ist im Vergleich mit den anderen Berufsgruppen am nächsten an den Patienten dran und hat die intensivsten Patientenkontakte. Sie besetzt eine zentrale Stellung im Versorgungsprozess und biopsychosoziales Handeln wie Trösten, Fördern oder Begleiten sind in der Tradition der Pflege tief verwurzelt. Aber auch in gruppentherapeutischen Maßnahmen ist Pflege immer wieder eingebunden. In einer Untersuchung konnten wir zeigen, dass Pflege in einer Klinik an 22,6\% der Gruppenangebote einer Klinik beteiligt war, während dies nur für 2,3\% Ärzte galt [9].

Gleichwohl weisen die Autoren zu Recht darauf hin, dass in der Pflege eine Differenzierung der Qualifikationsprofile nottut. Nicht ausgeschlossen, dass sich hier Arbeitsfelder für Menschen mit geringerer Qualifikation ergeben. Damit es aber Pflegende gibt, die im Rahmen eines komplexer werdenden Krankenhausalltags die Verantwortung für pflegerisches Handeln übernehmen können, sind Pflegeexperten mit Hochschulabschluss unabdingbar. Dies ist für den Leitungsbereich im Übrigen weitestgehend schon akzeptiert.

Eine Ausdifferenzierung pflegerischer Aufgaben sollte nicht nur mit den ärztlichen und psychologischen Bereichen abgestimmt werden, sondern mit allen anderen beteiligten Berufsgruppen (z.B. der Sozialarbeit). Außerdem können pflegerische Aufgaben nicht einfach in Grundpflege und spezielle Pflege aufgeteilt werden, wobei die Grundpflege von weniger qualifizierten Hilfskräfte, die spezielle Pflege aber von besonders ausgebildeten Pflegekräften übernommen würde. Einem Menschen bei der Körperpflege zu unterstützen kann eine einfache Tätigkeit sein, die von Hilfskräften durchgeführt werden kann, sie kann aber auch eine komplexe Pflegesituation darstellen, die die Anwesenheit der am besten ausgebildeten Pflegenden erfordert.

Vor dem Hintergrund, dass Personalabbau die Berufsgruppe der Pflege besonders getroffen hat, ist Delegation sicher nicht das Allheilmittel. Delegation meint ja die Verschiebung ärztlicher Tätigkeiten in die Pflege und nicht etwa umgekehrt. Dies bedeutet zunächst eine niedrigere Belastung für die Mediziner und eine Mehrbelastung der Pflege. Pflege übernimmt hier eine Lückenbüßerfunktion ohne den notwendigen Professionalisierungsschub.

Im täglichen Ringen um die Effizienzsteigerungen im Krankenhaus sieht sich die Pflege ständig Versuchen ausgesetzt, pflegefremde Tätigkeiten in den Stellenplan der Pflege zu verschieben (z.B. Stationssekretärin oder Servicekraft in den Stellenplan der Pflege). Der vorgestellten Differenzierung der Pflege kann man etwas abgewinnen, wenn z.B. Stationssekretärin und Servicekraft zusätzlich zum jetzigen Stellenplan gerechnet werden. Wenn diese Funktionen aber aus dem Stellenplan der Pflege kommen sollen, dann lässt sich das Konzept aus einem anderen Grunde nicht realisieren: Es gibt dann auf vielen Einheiten zu wenig Menschen, die mit dreijähriger Ausbildung alleine Nachtwachen machen können.

Aber was muss passieren? Mit Dahlgaard u. Stratmeyer [8] ist davon auszugehen, dass Rationalisierungspotenziale lediglich in einer berufsübergreifenden (kooperativen) Reorganisation der patientenbezogenen Leistungsprozesse liegt.

\section{Literatur}

1 Voderholzer $U$, Hohagen F Hrsg. Therapie psychischer Erkrankungen: State of the Art 2007/2008 (3. Auflage). München: Urban\& Fischer, 2007

2 Stellungsnahme der Bundesärztekammer und der Kassenärztlichen Bundesvereinigung. Dtsch Ärztebl 1988; 85: 2604-2605

3 Juckel G, Morosini PL. The new approach: psychosocial functioning as a necessary outcome criterion for therapeutic success in schizophrenia. Curr Opin Psychiatry 2008; 21 (6): 630-639

4 Amering M, Schmolke M. Recovery. Das Ende der Unheilbarkeit. Bonn: Psychiatrie Verlag, 2007

5 Weinmann S. Erfolgsmythos Psychopharmaka - Warum wir Medikamente in der Psychiatrie neu bewerten müssen. Bonn: Psychiatrie Verlag, 2008

6 Schulz M, Dorgerloh S, Ratzka S et al. Compliance und Adherence Therapeutische Interventionen zur Beeinflussung der Adherence chronisch Kranker gehören zur ureigensten Domäne professionellen pflegerischen Handelns. PADUA - die Fachzeitschrift für Pflegepädagogik 2007; 3: 44-49

7 Hausner H, Hajak G, Spießl H. „Kooperative Verantwortung“: Das neue Gutachten des Sachverständigenrates zur Entwicklung des Gesundheitswesens. Psychiat Prax 2007; 34: 355-366

8 Dahlgaard K, Stratmeyer P. Kooperatives Prozessmanagement im Krankenhaus. Das Krankenhaus 2004; (8): 634 -640

9 Schulz M, Renard C, Keogh J. Analyse des Gruppenangebotes einer psychiatrisch-psychotherapeutischen Klinik anhand von Struktur-, Prozess- und Ergebniskriterien. Krankenhauspsychiatrie 2006; 17: 25-30 